\title{
Ce que l'industrialisation a fait aux artisans d'Europe occidentale, années 1830 - années 1930
}

What industrialization did to the artisans of Western Europe, 1830s-1930s

\section{Cédric Perrin}

\section{(2) OpenEdition}

1 Journals

Édition électronique

URL : https://journals.openedition.org/artefact/6862

DOI : 10.4000/artefact.6862

ISSN : 2606-9245

Éditeur :

Association Artefact. Techniques histoire et sciences humaines, Presses universitaires de Strasbourg

\section{Édition imprimée}

Date de publication : 7 janvier 2021

Pagination : 317-334

ISBN : 978-2-8107-0706-5

ISSN : 2273-0753

Référence électronique

Cédric Perrin, «Ce que l'industrialisation a fait aux artisans d'Europe occidentale, années 1830 années 1930 », Artefact [En ligne], 13 | 2020, mis en ligne le 23 décembre 2020, consulté le 28 juin 2022. URL : http://journals.openedition.org/artefact/6862 ; DOI : https://doi.org/10.4000/artefact. 6862

\section{(c) (†) $९$}

Artefact, Techniques, histoire et sciences humaines est mise à disposition selon les termes de la Licence Creative Commons Attribution - Pas d'Utilisation Commerciale - Pas de Modification 4.0 International. 


\section{Ce que l'industrialisation a fait aux artisans d'Europe occidentale, années 1830 - années 1930}

\section{Cédric Perrin}

\section{Résumé}

La période qui s'étend des années 1830 aux années 1930 fut celle de l'industrialisation de l'Europe, qui fut longtemps interprétée comme une révolution industrielle dont les machines et les usines auraient condamné l'existence des artisans. Mais nous savons aujourd'hui que l'industrialisation ne fut pas cette révolution. Alors il faut revoir également ce qu'elle a pu faire aux artisans. L'article revient d'abord sur l'évolution de l'historiographie et son renouvellement. Puis une deuxième partie interroge ce qu'étaient le travail des artisans et ses transformations. Enfin, la dernière partie montre comment les artisans parvinrent, ou pas selon les pays, à s'organiser et à obtenir de leur État des institutions représentatives (l'handwerk en Allemagne, l'artisanat en France). L'industrialisation ne fit pas disparaître les artisans d'Europe occidentale mais elle transforma leurs métiers et leurs pratiques.

\section{Mots-clés}

artisan, artisanat, industrialisation, travail

95 Cédric Perrin, «Ce que l'industrialisation a fait aux artisans d'Europe occidentale, années 1830 - années $1930 »$, Artefact, 13, 2020, p. 317-334. 


\section{What industrialization did to the artisans of Western Europe, 1830s-1930s}

\section{Abstract}

The period from the 1830 s to the 1930 s was that of the industrialization of Europe, which has long been interpreted as an industrial revolution whose machines and factories would have condemned the existence of craftsmen. But we know today that industrialization was not this revolution. So we must also review what it did to the artisans. The article first discusses the evolution of historiography and its renewal. Then a second part questions what was the work of the craftsmen and its transformations. Finally, the last part shows how the craftsmen managed, or not according to the countries, to organize and obtain from their State representative institutions (handwerk in Germany, artisanat in France). Industrialization did not make the artisans of Western Europe disappear, but it transformed their trades and practices.

\section{Keywords}

craftsman, craft industry, industrialization, work 


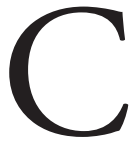

ette histoire pourrait être celle d'un malentendu. La " révolution industrielle ", par la force de ses machines et de ses usines, aurait voué la production artisanale à une inéluctable disparition. Pour parler des artisans, il ne resterait plus alors que le registre des lamentations ou de la nostalgie. Dans ses romans Colline (1929) et Regain (1930), Jean Giono, qui était le fils d'un cordonnier piémontais, célèbre une société de paysans et d'artisans qui s'évanouit. En Allemagne dès la fin du $\mathrm{XIX}^{\mathrm{e}}$ siècle, en France dans l'entre-deux-guerres, les États légiférèrent avec l'intention de protéger les artisans des menaces de l'économie industrielle afin de préserver l'équilibre social et politique. Mais l'industrialisation n'a pas été ce bouleversement soudain et radical que suppose le concept de révolution industrielle ${ }^{1}$. Dès lors, ne faudrait-il pas aussi reconsidérer les effets de l'industrialisation sur les artisans ? Est-il si sûr que les progrès de l'industrie ont fait disparaître ou pour le moins dépérir le monde des artisans ? Après tout, cette idée ne va-t-elle pas à l'encontre de nos vies quotidiennes, de l'expérience banale, par exemple, d'aller acheter du pain. Dans l'entre-deux-guerres, le nombre d'artisans varia peu en France ; en Suisse, il augmenta même sensiblement ${ }^{2}$. Si l'industrialisation n'entraîna pas la disparition des artisans, il serait tout aussi abusif de la considérer comme un processus neutre. Il faut donc repenser à nouveaux frais ce que l'industrialisation a fait aux artisans. Quelles réactions a-t-elle provoquées? Quelles transformations a-t-elle engagées? Comment les artisans se sont adaptés à la nouvelle organisation économique qui émergeait ? Ces pages ne sauraient apporter des réponses exhaustives et définitives à ces questions. Leur ambition est plus modestement d'ouvrir des pistes de réflexion et de poser quelques jalons sur celles-ci, à l'échelle de l'Europe occidentale.

\section{Regards sur les artisans}

Avec la révolution industrielle, les artisans parurent relever d'un passé révolu. L'artisanat devenait un sujet pour les médiévistes mais par pour le présent ; économistes et historiens contemporanéistes s'en détournèrent longtemps. Toutefois, depuis une quarantaine d'années, avec des hauts et des bas, petites entreprises et artisans retrouvent une place dans l'analyse de l'industrialisation.

\footnotetext{
1. Verley, 1997.

2. Laloire, 1955, p. 263 ; Perrin, 2007.
} 


\section{L'histoire du déclin et d'un oubli}

Au cours des années 1830, des observateurs s'enthousiasmèrent pour les nouveautés qui révolutionnaient, à leurs yeux, la production des industries. En 1837, l'économiste français Adolphe Blanqui employa l'expression de révolution industrielle qui allait connaître un durable succès ${ }^{3}$. Sur fond de croissance de la production, deux caractéristiques notamment retenaient l'attention et restèrent accolées à l'expression : la mécanisation et l'usine (le factory system) avec ses ouvriers dont la misère inquiétait. Une abondante littérature se développa ensuite sur ces thèmes du machinisme et de la " question sociale ". Ainsi posée, ladite révolution industrielle paraît condamner le travail des artisans. D'une part, le capital accumulé dans les machines surclasse les possibilités de production du travail manuel des artisans. D'autre part, ces artisans privés de travail n'ont plus d'autre avenir que d'aller grossir les rangs de la main-d'œuvre des usines. C'est probablement le philosophe allemand Karl Marx qui a le plus clairement exprimé cette conviction, notamment dans le Manifeste du parti communiste, rédigé avec Friedrich Engels, dans lequel les artisans sont promis à sombrer dans le prolétariat car leur habileté technique ne peut pas rivaliser avec la grande industrie capitaliste ${ }^{4}$.

Sans faire œuvre d'historiens, les économistes du XIX ${ }^{\mathrm{e}}$ siècle proposèrent des lectures par étapes de la croissance industrielle qui identifiaient le temps des artisans au passé. De même, pour les historiens allemands de la fin du siècle comme Karl Bücher, l'industrie capitaliste succéda au stade de la production artisanale et donc la dépassa. Le marxisme d'une part et l'école historique allemande d'autre part influencèrent durablement les historiens de la fin du XIX et du début du Xx ${ }^{e}$ siècles, britanniques et français notamment, et au-delà l'opinion publique et les dirigeants politiques.

Après la Seconde Guerre mondiale, les historiens s'intéressèrent très peu aux artisans. L'influence durable du marxisme orientait l'histoire sociale vers l'étude des classes sociales émergentes de la bourgeoisie industrielle et des ouvriers en délaissant les anciennes classes moyennes indépendantes - ou petite bourgeoisie - réputées sur le déclin, dont font partie les artisans. La forte croissance économique des Trente Glorieuses et l'importance

3. Verley, 1997, p. 23.

4. Marx, Engels, 1848, p. 15 ; Boutillier, 2015. 
accordée à la productivité et au fordisme incitaient les historiens de l'économie à en rechercher les débuts dans la naissance des grandes entreprises au XIX ${ }^{\mathrm{e}}$ siècle ${ }^{5}$ plutôt que dans les petites, a priori disqualifiées et déclinantes et pour lesquelles les sources paraissaient manquer. À l'inverse, l'artisanat paraissait trop lié aux régimes ruralisants et conservateurs de Vichy, Franco et Salazar et au retard économique. Le mouvement poujadiste passa pour la révolte contre son destin d'un groupe social sur le déclin.

\section{Un renouveau historiographique depuis les années 1970}

Les chocs économiques des années 1970 questionnèrent l'efficacité attribuée jusqu'alors à la grande grande entreprise fordiste tandis que l'influence sur les historiens des grands modèles idéologiques, dont le marxisme, marquait le pas. Ce nouveau contexte se traduisit par un regain d'intérêt des sciences sociales pour l'artisanat.

En 1975, la Commission internationale d'histoire des mouvements sociaux et des structures sociales choisissait la petite entreprise et la croissance industrielle pour thème de son congrès ${ }^{6}$. La revue Le Mouvement social y consacra deux numéros spéciaux en 1979 et 1981. Sous l'impulsion de Heinz-Gerard Haupt et Geoffrey Crossick, ces travaux prirent une dimension comparatiste à l'échelle de l'Europe (sans les pays méditerranéens toutefois) dans une perspective d'histoire politique et sociale du XIX ${ }^{\mathrm{e}}$ siècle $^{7}$. Un débat sémantique autour des notions de petite bourgeoisie ou de classes moyennes réactiva ceux de la fin du XIX ${ }^{\mathrm{e}}$ siècle. En effet, ce sont notamment les partis catholiques belges qui favorisèrent alors l'expression classes moyennes, reflet d'une société sans antagonismes sociaux. À l'encontre d'une lutte des classes, ces classes moyennes formeraient un point d'équilibre dans la société. Pour la France, les travaux de Serge Bernstein ont montré la place des artisans et des petits commerçants dans l'assise sociologique du Parti radical ${ }^{8}$. L'écho du poujadisme a conduit à scruter leur glissement à droite, voire un soutien aux fascismes en Italie puis en Allemagne. L'historiographie accorda aussi beaucoup d'importance aux

5. Fressoz, Jarrige, 2013.

6. Colloque, 1981.

7. Crossick, Haupt, 1984 et 1995.

8. Bernstein, 1980. 
sociabilités urbaines, aux pratiques culturelles et à la mobilité sociale 9 . Dans la même période, le concept de "production flexible " comme alternative à la production de masse soulignait les atouts et les capacités d'adaptation des entreprises artisanales et des $\mathrm{PME}^{10}$.

Depuis la fin des années 1990, les travaux de Steven Zdatny, puis les miens, se sont intéressés aux artisans en France au $\mathrm{Xx}^{\mathrm{e}}$ siècle ${ }^{11}$. Plusieurs auteures ont montré le rôle de la petite entreprise dans l'intégration des immigrants en France ${ }^{12}$. Il faut souligner l'apport des historiens modernistes qui ont montré, d'une part, que les corporations de métiers n’ont pas nécessairement été un carcan qui freinait l'innovation et ont, d'autre part, introduit une dimension anthropologique à l'étude du travail des artisans et de leur rapport aux techniques ${ }^{13}$. Sans prendre pour sujet les artisans, plusieurs études sectorielles et/ou régionales ont souligné leur place dans une industrialisation "douce ", voire "invisible ${ }^{14}$. Les petites entreprises des artisans font partie de la "face cachée " de l'industrialisation espagnole ${ }^{15}$ et des districts industriels de la "troisième Italie ${ }^{16}$. Enfin, les travaux sur l'aryanisation économique, c'est-à-dire le vol des entreprises dites juives durant la Seconde Guerre mondiale, dont les petits entrepreneurs furent les plus nombreuses victimes, ont également mis en lumière l'importance de l'artisanat dans de nombreux secteurs avant le conflit ${ }^{17}$.

\section{Un travail en mutations}

\section{Hommes de métier et métiers d'artisans}

Au $\mathrm{Xx}^{\mathrm{e}}$ siècle, l'artisan est un petit producteur indépendant mais ce dernier critère ne s'imposa que progressivement au cours du XIX ${ }^{e}$ siècle $^{18}$. Dans les langues italienne, espagnole et française, la racine latine (ars) rappelle

\footnotetext{
9. Guillaume, 1995.

10. Sabel, Zeitlin, 1985 ; Piore, Sabel, 1989.

11. Zdatny, 1999.

12. Bruno, Zalc, 2006 ; Zalc, 2010 ; Martini, 2016.

13. Hilaire-Perez, 2013 ; Nègre, 2016 ; Cummins, Ó Gráda, 2019.

14. Olivier, 2004 ; Minovez, 2012.

15. Catalan, Nadal, 1994.

16. Bagnasco, 1977, Becattini, 1979.

17. Bajohr, 2002 ; Le Bot, 2007.

18. Perrin, 2020.
} 
que l'artisan était d'abord celui qui maîtrise son art, les arts mécaniques ; l'homme de métier. Dans les dictionnaires de Laveaux (1820) et de Poitevin (1856), il se distinguait de l'ouvrier par sa qualification, non par l'indépendance. Avec le louage d'ouvrage et le tâcheronnage, l'ouvrier n'était pas, quant à lui, forcément un salarié ${ }^{19}$. Des catégories intermédiaires entre l'indépendance et le salariat et une certaine fluidité des statuts existaient avec des individus qui évoluaient de l'un à l'autre. De plus, les artisans pouvaient avoir plusieurs activités, notamment en milieu rural.

Dès lors, les dénombrer est malaisé pour le XIX siècle. En Angleterre, où le statut d'artisan n'a pas de fondement juridique, cela reste impossible. Les statistiques industrielles ne retenaient souvent que les établissements importants. En Belgique et en France, la croissance des patentés entre 1870 et 1914, plus rapide que celle de la population, a fait apparaître cette période comme un âge d'or des petits patrons, qui se retrouve aussi en Allemagne. En 1906, les établissements de moins de dix ouvriers représentaient $58 \%$ de la population industrielle active française. Dans les recensements industriels italiens de 1927 et 1937, 35\% des entreprises d'au moins un salarié en occupaient entre un et neuf. Mais ces données mesurent des catégories plus larges que celle d'artisan. Les chiffres plus précis sont tardifs (tableau 1).

Tableau 1 - Les artisans à la fin des années 1930

\begin{tabular}{|l|l|l|}
\hline \multicolumn{1}{|c|}{ Pays } & \multicolumn{1}{c|}{ Effectifs } & \multicolumn{1}{c|}{ Années } \\
\hline France & 735000 & 1938 \\
\hline Allemagne & 793000 & 1939 \\
\hline Belgique & 164500 & 1937 \\
\hline Luxembourg & 6700 & 1939 \\
\hline
\end{tabular}

Sources : Laloire, 1955 ; Perrin, 2007.

Que les artisans furent menacés par l'industrie suppose que les deux se concurrencèrent. Or, ce n'est que partiellement vrai. Trois branches concentrèrent les artisans jusqu' au milieu du $\mathrm{xx}^{\mathrm{e}}$ siècle : le bâtiment, l'alimentation et la confection. Le bâtiment (avec ses maçons, charpentiers, peintres, menuisiers...), comme la boulangerie et la boucherie, reste durablement dominé par l'artisanat. Vers 1900, près de $93 \%$ de ses entreprises

19. Didry, 2016 ; Martini, 2019. 
ont moins de dix salariés en France ${ }^{20}$. Jusque vers 1880, les problèmes de transport ont maintenu une structure atomisée de petits producteurs qui fournissaient des marchés locaux dans la meunerie, les brasseries et les huileries. En Espagne, en dehors de la Catalogne, moudre était alors la principale activité industrielle et la majorité des moulins n'était exploitée qu'une partie de l'année par des meuniers-paysans ${ }^{21}$. Leur nombre diminua ensuite mais l'alimentation resta cependant marquée par le minifundismo, la très petite taille des entreprises. Des artisans produisaient la majorité du sucre des Canaries, par exemple, et la tentative d'y développer un établissement industriel dans les années 1920 échoua $^{22}$.

Le cas du textile est intéressant car il inspira Engels à partir de ce qu'il en observa à Manchester. Indiscutablement, l'industrialisation du textile proprement dit (filature, tissage, impression) marginalisa les artisans tisserands mais la confection, avec ses tailleurs et ses couturières, demeurait artisanale. À peine $4 \%$ des tailleurs anglais et gallois employaient plus de dix personnes en $1851^{23}$. La confection rassemblait, avec les chaussures, près de la moitié des artisans belges en $1896^{24}$.

324 L'industrialisation fit davantage sentir ses effets dans les autres activités : travail des métaux, du cuir, du bois ou encore l'imprimerie. Les artisans, peu nombreux, s'y sont maintenus sur des segments de marché (luxe, produits d'art...), dans des fabrications encore peu standardisées (ébénistes, tapissiers...) et dans des territoires spécialisés, dont les districts industriels particulièrement importants en Italie et en Espagne ${ }^{25}$ mais également présents plus au nord avec par exemple les couteliers de Sheffield, de Solingen, de Thiers et de Nogent-en-Basigny.

Viennent enfin les services, sous-estimés par une historiographie plus attentive aux industries mais en essor avec les métiers de la coiffure, de l'entretien et de la réparation. Celui de cordonnier glissa de la fabrication vers la réparation. Dans l'automobile, hormis les premières années (mais ce n'est pas anodin dans le rapport aux innovations), les artisans étaient absents de la fabrication mais cette industrie n'aurait pas pu se développer

20. Lescure, 1983.

21. Nadal, 1992.

22. Luxán, Bergasa, 2001.

23. Verley, 1997, p. 153.

24. Kurgan-Van Hentenryk, 1984.

25. Catalan, Miranda, Ramon-Muñoz (éd.), 2011. 
sans le réseau des artisans garagistes qui entretenaient et réparaient les véhicules. Ainsi, si l'industrialisation réduisit certains métiers artisanaux, elle en fit aussi apparaître de nouveaux qui ont permis son renouvellement et sa pérennité.

\section{Le genre du travail et le travail familial}

L'artisanat est un milieu professionnel très genré. Peu de ces hommes de métier étaient des femmes; seulement $20 \%$ selon les registres des métiers ouverts en France en 1936. Les métiers du bâtiment sont même quasi exclusivement masculins. Les artisanes se concentraient dans l'entretien et la fabrication des vêtements (lingère, couturière, modiste...), où elles étaient nettement majoritaires, et dans la coiffure dont elles ont contribué à l'essor, notamment dans l'entre-deux-guerres. Quelques-unes exerçaient des métiers aux caractéristiques techniques proches (tapissières, bourrelières...). Les préjugés sociaux, reproduits par l'enseignement ${ }^{26}$, cantonnaient les femmes dans les métiers d'aiguille réputés féminins.

Les études sur les entreprises familiales se sont développées mais elles concernent le plus souvent de grandes entreprises ${ }^{27}$. La majorité des artisans, à l'image du menuisier Joachim Martin ${ }^{28}$, travaillaient seuls, sans ouvrier, ni leur épouse ou enfants. En 1895, 60 \% des artisans berlinois travaillaient seuls; proportion qui se retrouve parmi les artisans français dans les années $1930^{29}$. L'activité de l'artisan ne suffisait pas à dégager deux salaires et les épouses avaient leur emploi salarié à l'extérieur. Dans la Catalogne de la fin du XIX ${ }^{e}$ siècle, elles étaient ouvrières dans les usines textiles ${ }^{30}$.

Le travail familial était une réalité mais, le plus souvent, occasionnelle et ponctuelle, avec de sensibles variations selon les activités. Il relevait de la logique du coup de main, pas toujours rémunéré. L'apport en travail des épouses, qui aidaient à rédiger les factures sur leur temps libre, par exemple, se trouve ainsi difficile à mesurer et en partie masqué. Le travail familial était plus fréquent dans les métiers de l'alimentation où l'atelier est associé

26. Voir l'article de Stéphane Lembré dans ce volume.

27. Colli, Rose, 2008.

28. Boudon, 2017. Sur les pays germaniques : Beck, 2019.

29. Blackbourn, 1984, p. 40 ; Perrin, 2007.

30. Enrech, 2005. 
à une boutique qui est tenue par l'épouse. Surtout, il était plus souvent mobilisé par les façonniers, notamment dans le textile et le bâtiment, qui se situaient à la frange du salariat et de l'artisanat, catégories elles-mêmes en construction, on l'a vu, au tournant des deux siècles ${ }^{31}$.

La famille pose aussi la question de l'héritage et de la transmission. Or, l'artisanat est marqué par un fort brassage. À Bruxelles, seuls les plus gros patentés transmettaient leur entreprise, pas les artisans. Les dynasties d'artisans restèrent rares. Peu d'artisans sont des héritiers. Ils ont créé euxmêmes leur activité. Jusque dans les années 1930, l'artisan était d'abord un ouvrier qui se mettait à son compte pour travailler pour lui-même et être son propre patron. Ce renouvellement permanent fut aussi une condition qui assura la pérennité de l'artisanat.

\section{Du travail manuel et de la mécanisation}

L'industrialisation conduisit à caractériser le travail de l'artisan en travail manuel. C'est explicite dans les langues allemande (handwerker) et anglaise (handicraftsman). Le machinisme apparut comme un péril qui obscurcissait l'avenir des artisans, particulièrement celui des tisserands ${ }^{32}$. Mais la dimension manuelle du travail artisanal n'implique pas qu'il soit resté figé et étranger aux évolutions techniques. Il mua avec l'industrialisation et la mécanisation.

Par rejet de la modernité, les mouvements conservateurs enfermèrent les artisans dans une idéalisation restrictive et archaïsante du travail manuel qui les empêchèrent, là où ils accédèrent au pouvoir, d'en comprendre les besoins et les enjeux ${ }^{33}$. Mais l'artisanat n'est pas ce conservatoire des techniques préindustrielles. La mécanisation recouvre des réalités fort diverses qui ne se limitent pas à l'automation et aux installations industrielles lourdes et gourmandes en capitaux. Elle se démultiplia aussi en de nombreuses petites machines relativement peu coûteuses et faciles à installer ; certaines ne requéraient aucune source d'énergie. Les premières machines à coudre étaient mises en mouvement avec une pédale, par exemple. À partir de la fin du XIX ${ }^{\mathrm{e}}$ siècle, les nouvelles énergies facilitèrent la diffusion

31. Martini, 2017, p. 97.

32. Sennett, 2010.

33. Perrin, 2017. 
de machines et d'outils mus par de petits moteurs thermiques et surtout électriques. Diverses archives attestent de la pénétration des machines dans les ateliers artisanaux : les installations déclarées par des artisans dans les dossiers d'établissements dangereux, les en-têtes de leurs courriers où ils affichent fièrement leurs machines, les publicités des fabricants dans la presse professionnelle... Un véritable marché du matériel professionnel pour les artisans s'est développé.

L'introduction de ces machines transforma les procédés de fabrication artisanaux et le savoir opérationnel des artisans ${ }^{34}$. Savoir se servir de ces équipements entra dans la maîtrise de son art par l'homme de métier. Elle induisit aussi de nouveaux gestes techniques et de nouveaux rapports des corps au travail. Le travail artisanal est un travail physiquement pénible qui éprouve les corps. Les artisans et leurs ouvriers mettent en œuvre des matières malodorantes, voire toxiques, manipulent des charges lourdes, utilisent des machines dangereuses qui peuvent blesser et mutiler.

Enfin, la mécanisation n'eut pas des conséquences uniformes et linéaires. L'artisanat rural, par exemple (avec ses maréchaux, charrons, bourreliers...) profita d'abord des transformations de l'agriculture dont il accompagna, et permit, la mécanisation. Puis le remplacement de la traction hippomobile par l'automobile le fit reculer. En revanche, cette même évolution technique, de même que l'électricité, donna naissance à de nouveaux métiers : les garagistes, on l'a vu, mais aussi les taxis par exemple. Les photographes également exercent un métier nouveau qui repose sur l'usage d'une (petite) machine.

\section{Un monde qui s'organise}

\section{L'organisation de l'artisanat allemand}

Dès la fin du XVIII ${ }^{\mathrm{e}}$ siècle, la moitié des artisans des pays allemands exerçaient hors de toute corporation. Lexpansion du Code civil avec les armées françaises ne porta un coup qu'à une institution déjà moribonde et qui continua de décliner au début du $\mathrm{XIX}^{\mathrm{e}}$ siècle. Les lois de 1867 et 1869 sur le commerce et l'industrie de la Confédération germanique

34. Schwint, 2002. 
consacrèrent l'évolution libérale en enlevant tout pouvoir aux corporations qui subsistaient ${ }^{35}$.

Cependant, après les révolutions de 1848 qui avaient fait vaciller leurs trônes, les princes allemands cherchaient à détacher les artisans du mouvement ouvrier. Pour conduire sa politique anti-socialiste, le chancelier Bismarck rechercha le ralliement de l'aile corporatiste et conservatrice des artisans, que la grande dépression malmenait. À partir de 1881, de nouvelles corporations purent être créées dont les pouvoirs, notamment en matière d'apprentissage, furent progressivement élargis. La loi du 26 juillet 1897 créa des Chambres de métiers qui chapeautaient toutes les corporations d'une région ; ce qui était entièrement nouveau. Le régime nazi centralisa ensuite cette organisation sous la direction d'un reichhanwerksmeister.

Cette législation n'établit pas de définition officielle de l'artisan. Celle-ci reposait sur la jurisprudence qui mettait en avant la qualification, sans référence à la taille de l'entreprise. Elle favorisait ainsi la domination des petits patrons, suscitant les critiques des socialistes à l'encontre de ces « soi-disant artisans qui emploient 20 à 50 travailleurs ${ }^{36}$. Ces notables mobilisèrent un répertoire d'inspiration médiévale (blasons de métiers, titres...) pour inventer la tradition dont ils revendiquaient l'héritage ${ }^{37}$. Mais, en pensant faire renaître les anciennes corporations, c'est bien une institution nouvelle - l'artisanat (handwerk) - qu'ils créaient ainsi.

\section{La naissance de l'artisanat en France et ses débats}

L'Alsace-Lorraine disposait de sa Chambre de métiers quand elle fit retour à la France en 1918. Ses dirigeants militèrent pour conserver leur institution et la présenter comme un modèle à étendre aux autres régions françaises. Le mot artisanat entra dans le vocabulaire français à cette occasion. De fait, il existait bien quelques chambres de métiers en France avant la guerre mais il s'agissait d'associations d'orientation professionnelle. Les artisans français étaient représentés par des syndicats de métier. Au lendemain de la guerre, deux cordonniers parisiens, Robert Tailledet et Georges

35. Schriewer et Harney, 1999.

36. Sinner, 1981, p. 110.

37. Hobsbawm et Ranger, 2006. 
Grandadam, encouragés par le sénateur radical Étienne Clémentel, entreprirent de rassembler tous les artisan ${ }^{38}$. Les représentants alsaciens se joignirent à eux pour fonder en 1922 la Confédération générale de l'artisanat français (CGAF). Les Chambres de métiers furent créées en France avec la loi, dite Courtier, de 1925. Elles représentaient directement les artisans, sans que ne fussent recréées des corporations, à la différence du modèle allemand.

Les Alsaciens quittèrent la CGAF dès 1924 pour fonder une organisation concurrente. Cette scission est révélatrice des débats qui marquèrent la naissance de l'artisanat en France au sein duquel deux grands courants virent le jour : l'un dit syndicaliste, plus à gauche ; l'autre corporatiste et plus à droite.

Au-delà de divergences sur la stratégie et les finalités de l'artisanat, ils s'opposaient sur la définition même de l'artisan ${ }^{39}$. Pour les syndicalistes de la CGAF, l'artisan devait participer habituellement et personnellement à la production aux côtés de ses ouvriers, quand il en avait. Cela supposait de limiter la main-d'œuvre à un seuil au-delà duquel l'artisan devient un petit patron qui ne s'occupe plus que de la gestion. Les corporatistes refusaient ce plafond qui les exclurait eux-mêmes et qu'ils jugeaient arbitraire car pour eux c'est la qualification qui fait l'artisan; ils restaient fidèles en cela à l'héritage allemand. Alors qu'ils restaient proches des organisations de classes moyennes et du petit patronat, la CGAF affirmait l'autonomie de l'artisanat et en obtenait la reconnaissance par l'État à travers des lois sur la fiscalité, le crédit, l'apprentissage ou encore la création du registre des métiers pour lequel un seuil de cinq salariés fut retenu. L'artisanat s'organisa donc en France en suivant une voie différente de celui d'Allemagne. De telles différences se retrouvent à l'échelle de l'Europe.

\section{Prolongements européens}

Bien que proche des partis de gauche, Robert Tailledet n'hésita pas à se rapprocher du fasciste Vincenzo Buronzo, président de l'Ente Nazionale per l'Artigianato e le Piccole Industrie, pour créer un Institut international de l'artisanat dont le premier congrès se déroula à Rome en 1930. Le

38. Kessler et Rousseau, 2018.

39. Zarca, 1986. 
fascisme engagea bien quelques mesures favorables à l'artisanat, comme le financement du crédit artisanal en 1937, mais favorisa davantage les grandes entreprises capitalistes. Toutefois, l'organisation corporative de sa Carta del lavoro de 1927 inspira ensuite le Fuero del trabajo qui mit en place le syndicalisme vertical de l'Espagne franquiste en 1938. L'artisanat espagnol fut placé sous la direction d'un syndicat unique, la Obra sindical de artesania. Sa définition s'inspira de la législation allemande en ne retenant pas de limite de taille mais en ne reconnaissant comme artisanaux que les métiers d'art.

L'institut international créé par Tailledet et Buronzo naquit en se séparant de la Confédération internationale des classes moyennes dont le siège était à Bruxelles. En effet, la Belgique, dans le contexte de la Grande dépression, joua un rôle actif dans l'émergence de la notion de classes moyennes, réunissant artisans, commerçants et petits industriels. En 1896, une commission des classes moyennes fut créée au ministère de l'Industrie et du Commerce. Toutefois, attaché au libéralisme économique, le gouvernement catholique belge refusa d'intervenir directement, par une organisation publique de l'artisanat. De même, l'opposition à l'intervention de l'État était fortement ancrée au Royaume-Uni où aucune initiative ne fut prise pour créer des institutions équivalentes aux chambres de métiers allemandes ou françaises ${ }^{40}$.

Entre 1830 et 1940, l'industrialisation a eu sur les artisans des effets variés selon les activités, les périodes et les lieux mais elle ne les a pas fait disparaître. Les branches où les artisans étaient nombreux sont restées faiblement concentrées et inversement. La concurrence fut limitée entre la grande industrie et l'artisanat qui paraissent plus complémentaires que concurrents. La mécanisation n’est pas le seul déterminant de ces évolutions ; l'état des marchés (transport, nature de la demande...) est au moins aussi important. En revanche, les mutations techniques qui marquent le siècle de l'industrialisation ont aussi transformé les modalités pratiques du travail des artisans. Enfin, bien qu'il soit fréquent de présenter les organisations contemporaines dans la lignée des anciennes corporations, il n'existe pas de liens entre elles. L'apparition de nouvelles catégories de production et de producteurs redéfinit ce que sont les artisans et incita ceux-ci à

40. Crossick et Haupt, 1984, p. 260. 
s'organiser. Alors que l'industrialisation a paru les menacer, elle a eu pour effet paradoxal la naissance de l'artisanat ; l'expression elle-même apparut dans cette période.

\section{Bibliographie}

Bagnasco Arnaldo, Tre Italie: la problematica territoriale dello sviluppo italiano, Il Mulino, Bologne, 1977.

BajoHr Frank, Ayranisation in Hamburg, The economic exclusion of Jews and the confiscation of their property in nazi germany, Berghahn Books, New York, 2002.

Becattini Giacomo, "Dal settore industriale al distretto industriale: alla ricerca dell'unita d'indagine della economia industriale ", Revista di economia e politica industriale, $\mathrm{n}^{\circ} 1,1979$, p. 7-21.

BECK Robert, Dieu, le roi et la bière. Un artisan bavarois (1821-1872), Presses universitaires François-Rabelais, Tours, 2019.

Bernstein Serge, Histoire du parti radical, PFNSP, Paris, 1980.

Blackbourn David, "Between resignation and volatility: the German petite bourgeoisie in the nineteenth century ", dans Crossick Geoffrey, HaUpt Heinz-Gerard (dir.), Shopkeepers and masters artisans in Nineteenth century Europe, Methuen, Londres/New York, 1984, p. 35-61.

Boudon Jacques-Olivier, Le Plancher de Joachim. L'histoire retrouvée d'un village français, Gallimard, Paris, 2017.

Boutillier Sophie, «Le devenir de l'artisanat au XIx ${ }^{e}$ siècle, évolution des métiers dans le développement du capitalisme ", Marché et organisations, ${ }^{\circ} 24,2015$, p. 201-217.

Bruno Anne-Sophie, Zalc Claire, Petites entreprises et petits entrepreneurs étrangers en France (XIX'-XX' siècle), Publibook, Paris, 2006.

Catalan Jordi, Miranda José, Ramon-Muñoz Ramon (dir.), Distritos y clusters en la Europa del sur, Lid, Madrid, 2011.

Catalan Jordi, NaDAl Jordi (dir.), La cara oculta de la industrialización española. La modernización de los sectores no líderes (siglo XIX y XX), Alianza, Madrid, 1994.

Colli Andrea, Rose Mary, "Family Business", dans Jones Geoffrey, Zeituin Jonathan (dir.), The Oxford Handbook of Business History, Oxford University Press, Oxford, 2008, p. 194-218.

Colloque, Petite entreprise et croissance industrielle (XIX'-XX' siècle), CNRS, Paris, 1981.

Cummins Neil, Ó Gráda Cormac, Artisanal Skills, Watchmaking, and the Industrial Revolution: Prescot and Beyond, Working papers series, $\mathrm{n}^{\circ} 440$, University of Warwick, octobre 2019. 
Crossick Geoffrey, Haupt Heinz-Gerard (dir.), Shopkeepers and masters artisans in Nineteenth century Europe, Methuen, Londres/New York, 1984.

Crossick Geoffrey, Haupt Heinz-Gerard (dir.), The petite bourgeoisie in Europe 1780-1914, Routledge, Londres/New York, 1995.

Didry Claude, L'Institution du travail. Droit et salariat dans l'histoire, La Dispute, Paris, 2016.

Enrech Carles, "Hiérarchisation de l'usine et qualification dans l'industrie textile catalane du dernier tiers du XIX siècle ", Histoire et Sociétés, n 14, 2005, p. $10-23$.

Fressoz Jean-Baptiste, JARRIge François, "L'histoire et l'idéologie productiviste. Les récits de la "révolution industrielle" après 1945 ", dans PEssis Céline, Topçu Sezin, Bonneuil Christophe (dir.), Une autre histoire des Trente Glorieuses : modernisation, contestations et pollutions dans la France d'après-guerre, La Découverte, Paris, 2013, p. 61-79.

Guillaume Pierre (dir.), Regards sur les classes moyennes XIX $-X X^{e}$ siècles, EMSHA, Talence, 1995.

Hilaire-PÉrez Liliane, La Pièce et le geste : Artisans, marchands et savoir technique à Londres au XVIII siècle, Albin Michel, Paris, 2013.

Hobsbawm Eric et Ranger Terence (dir.), L'Invention de la tradition, Éditions Amsterdam, Paris, 2006.

Kessler Marie-Christine, Rousseau Guy (dir.), Étienne Clémentel. Politique et action publique sous la Troisième République, Peter Lang, Bruxelles, 2018.

Kurgan-Van Hentenryk Ginette, "A forgotten class: the petite bourgeoisie in Belgium, 1850-1914 ", dans Crossick Geoffrey, Haupt Heinz-Gerard (dir.), Shopkeepers and masters artisans in Nineteenth century Europe, Methuen, Londres/New York, 1984, p. 120-136.

LALOIRE Marcel, "Problèmes de l'artisanat européen ", Revue internationale du travail, $\mathrm{n}^{\circ} 72,1955$, p. 260-283.

Le Bot Florent, La Fabrique réactionnaire. Antisémitisme, corporatisme et spoliations dans le cuir, Presses de Sciences Po, Paris, 2007.

Lescure Michel, Histoire d'une filière : immobilier et bâtiment en France (18201980), Hatier, Paris, 1983.

Luxán Santiago de, Bergasa Oscar, "Un experimento fallido de industrialisación : tapices y fábricas de azúcar en Canarias, 1876-1933 ", Revista de estudios regionales, $\mathrm{n}^{\circ} 60,2001$, p. 45-78.

Martini Manuela, "Tâcherons ou sous-traitants ? Travailleurs indépendants et entrepreneurs dans la construction en France entre la fin du XIX ${ }^{\mathrm{e}}$ siècle et l'entre-deux-guerres ", Revue de synthèse, $\mathrm{n}^{\circ}$ 4, 2019, p. 43-84.

Martini Manuela, "Migrations et travail en famille. Recenser les entreprises familiales des maçons migrants en banlieue parisienne entre la fin du XIX et 
le début du $\mathrm{xx}^{\mathrm{e}}$ siècle ", dans Dubert Isidro, Gourdon Vincent (dir.), Inmigración, trabajo y servicio doméstico: En la Europa urbana, siglos XVIII-XX, Casa de Velázquez, Madrid, 2017, p. 93-108.

Martini Manuela, Le Bâtiment en famille. Migrations et petite entreprise en banlieue parisienne au XXe siècle, CNRS Éditions, Paris, 2016.

Marx Karl, Engels Friedrich, Manifeste du parti communiste, Livre de poche,

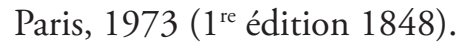

Minovez Jean-Michel, L'Industrie invisible : les draperies du Midi, XVIT -XX Essai sur l'originalité d'une trajectoire, CNRS Éditions, Paris, 2012.

NADAL Jordi, Moler, tejer y fundir. Estudios de historia industrial, Ariel, Barcelone, 1992.

NÈGRe Valérie, L'Art et la matière. Les artisans, les architectes et la technique (17701830), Garnier, Paris, 2016.

Olivier Jean-Marc, Des clous, des horlogers et des lunettes. Les campagnards moréziens en industrie (1780-1914), CTHS, Paris, 2004.

Perrin Cédric, "Parole in storia: Artigiano/Artisan ", Diacronie, n² 22, 2020, en ligne: https://www.studistorici.com/2020/04/30/parole-in-storia-artisan-fr/.

Perrin Cédric, "Un ordre économique sans machine ? Penser et repenser l'artisanat et la société française au Xx $x^{\mathrm{e}}$ siècle ", L'Homme et la Société, nº 205, 2017, p. 185-209.

Perrin Cédric, Entre glorification et abandon. L'État et les artisans en France (19381970), CHEFF, Paris, 2007.

Piore Michael, SABel Charles, Les Chemins de la prospérité : de la production de masse à la spécialisation souple, Hachette, Paris 1989.

Sabel Charles, Zeituin Jonathan, "Historical Alternatives to Mass Production: Politics, Markets, and Technologies in Nineteenth-Century Industrialization ", Past \& Present, $\mathrm{n}^{\circ}$ 108, 1985, p. 133-176.

Schriewer Jürgen, Harney Klaus, "Beruflichtkeit versus culture technique. Contribution à la sémantique du travail en France et en Allemagne ", dans Zimmermann Bénédicte, Diddry Claude et Wagner Peter (dir.), Le Travail et la nation. Histoire croisée de la France et de l'Allemagne, Éditions de la Maison des Sciences de l'Homme, Paris, 1999, p. 107-146.

Schwint Didier, Le Savoir artisan. L'efficacité de la mètis, L'Harmattan, Paris, 2002.

Sennett Richard, Ce que sait la main. La culture de l'artisanat, Albin Michel, Paris, 2010.

SinNer Eugen, "La politique de la social-démocratie allemande vis-à-vis de l'artisanat à la fin du XIX e siècle ", Le Mouvement social, n 114, 1981, p. 105-123.

VerLey Patrick, L'Échelle du monde. Essai sur l'industrialisation de l'Occident, Gallimard, Paris, 1997. 
Cédric Perrin

Zalc Claire, Melting shops, Une histoire des commerçants étrangers en France, Perrin, Paris, 2010.

ZARCA Bernard, L'Artisanat français, du métier traditionnel au groupe social, Economica, Paris, 1986.

Zdatny Steven, Les Artisans en France au XX' siècle, Belin, Paris, 1999.

\section{L'auteur}

Cédric Perrin est professeur agrégé, docteur en histoire et chercheur rattaché à l'IDHE.S Évry. Il travaille sur l'histoire des artisans, des petites entreprises et de l'apprentissage. Il a notamment publié : Entre glorification et abandon. L'État et les artisans en France (1938-1970), CHEFF, 2007 et dirigé, avec S. Boutillier et C. Fournier, « Le temps des artisans. Permanence et mutations », Marché \& Organisations, $n^{\circ} 24,2015$. Contact : cp2002@orange.fr 